\title{
Lumbar Total Disc Replacement defeats crippling sciatica
}

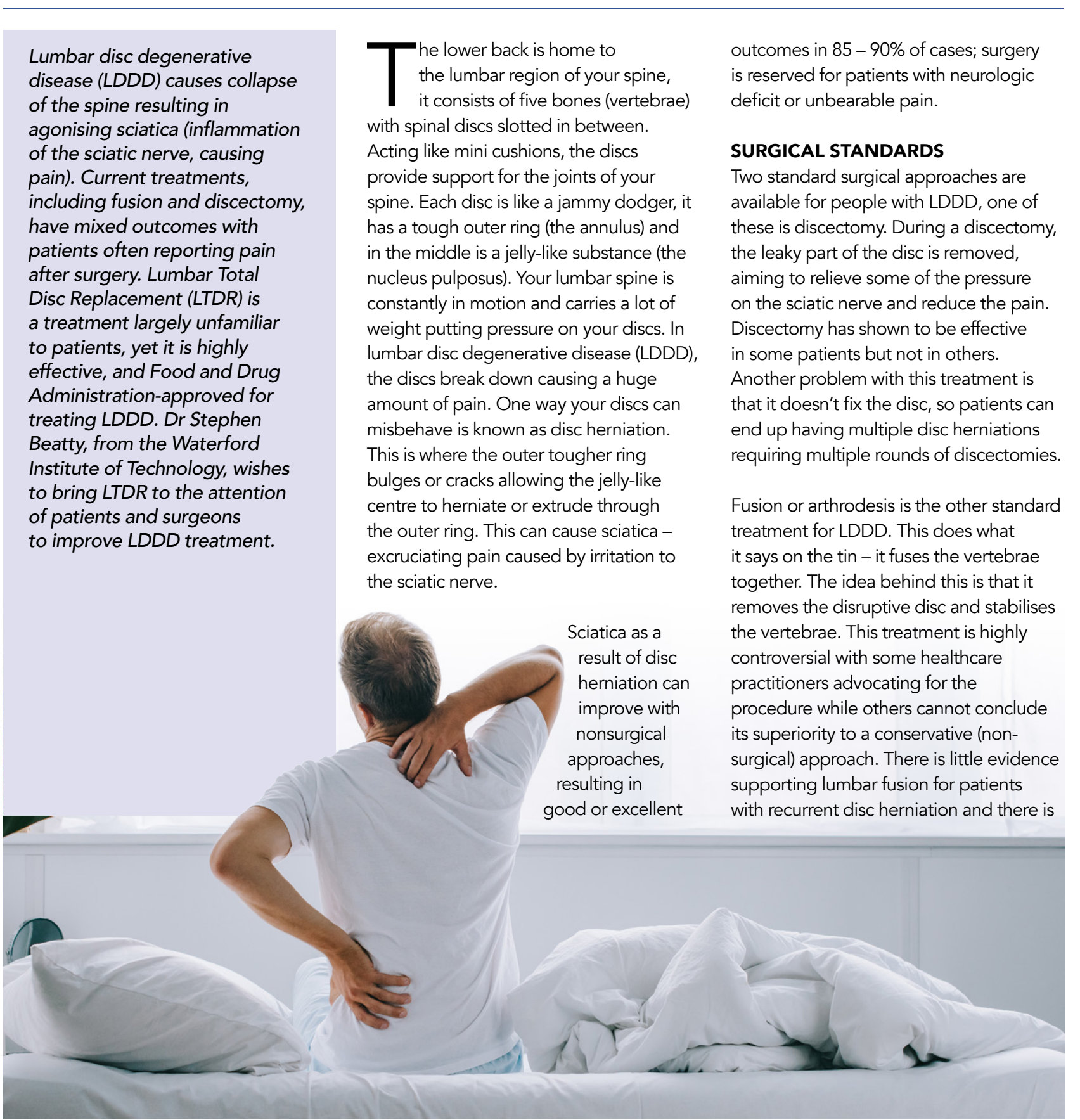

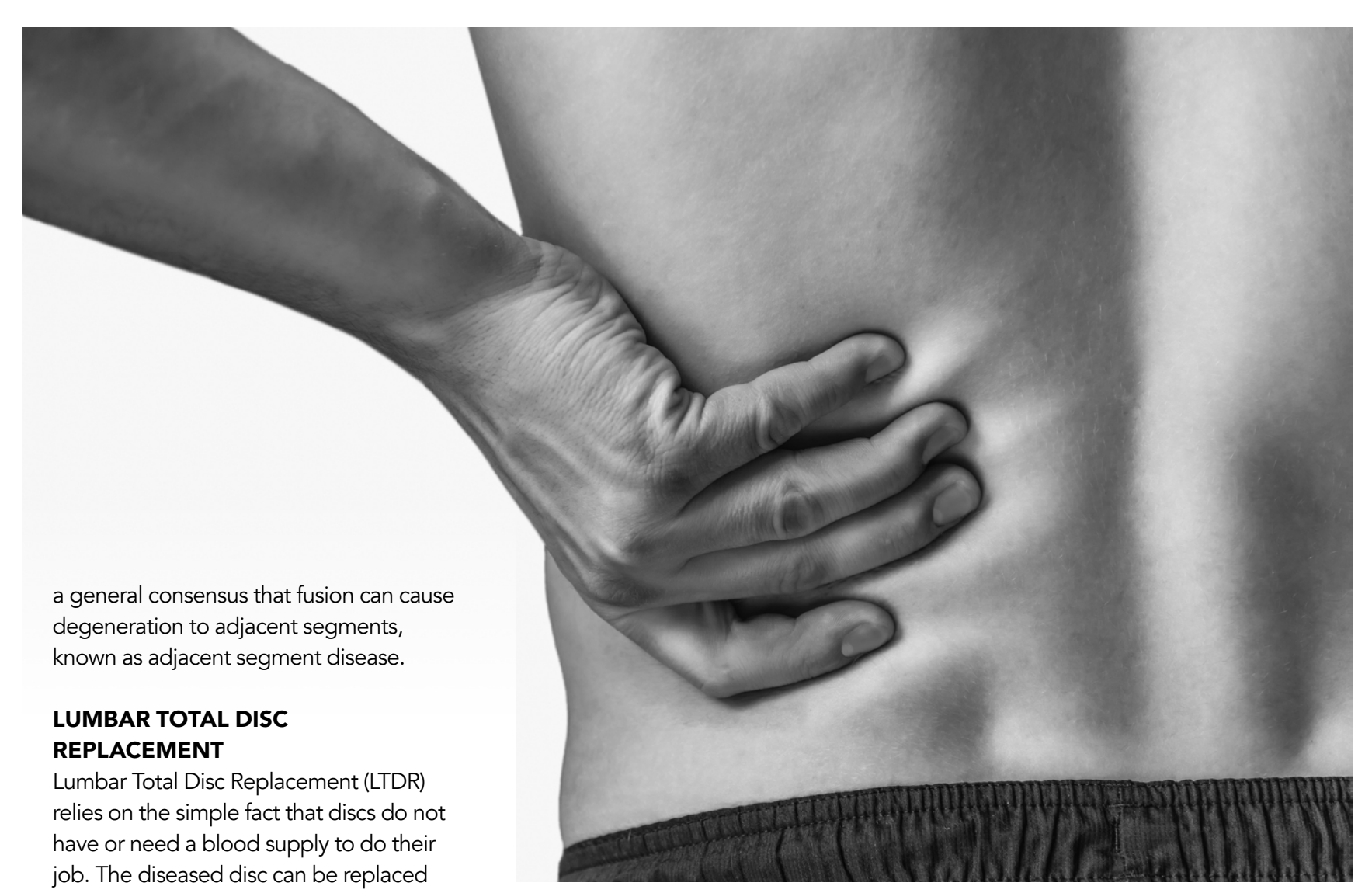

job. The diseased disc can be replaced with a lumbar artificial disc (LAD), relieving the patient of sciatica, reducing the risk of adjacent segment disease and allowing the patient to maintain the normal physical movements of the spine. This sounds like a breakthrough, so what's the problem?

The clinical use of LADs in LTDR has been somewhat frustratingly slow and steady. The first $L A D$, a steel ball, was implanted in the 1960 . Complications after surgery prompted rethe 1900 s of the technique Encourat the $190 \mathrm{l}$ and teally $2000 \mathrm{~s}$. 1990s, emerging from small amounts of evidence, paved the way for ITDR, resulting in the uptake of the procedure by many spinal surgeons and medical device companies. Due to its complex and technically demanding nature, spinal surgeons continue to adopt LTD but slowly.

To date, the most successful LAD is ProDisc-L, based on a ball and socket principle made with cobalt and polyethylene. It has been rigorously tested in clinical trials with astonishing results. In 2007, the US Food and Drug Administration (FDA) compared outcomes following LTDR (161 patients) verses fusion
treatment (75 patients) in a randomised,

Due to its complexities and technically demanding nature, spinal surgeons continue to adopt LTDR; but slowly. controlled trial. The researchers found after two years the LTDR group fared better in terms of pain, patient satisfaction and range of movement when compared

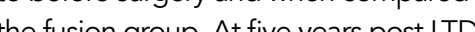
the fusiongroup. At five years post Interestingly, this study wasn't designed to show a difference between these two techniques, its intention was to demonstrate that LTDR wasn't worse than the standard fusion treatment (these are called noninferiority trials and are

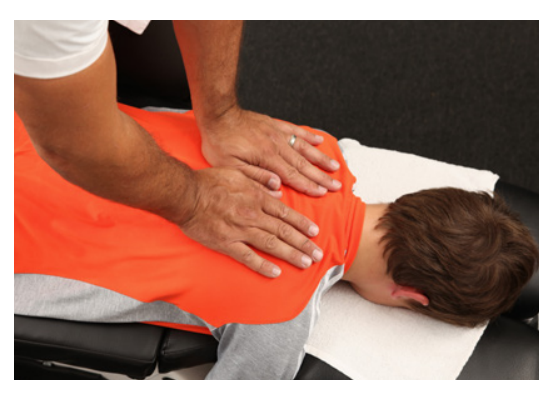
Sciatica a a a result of dischernial
with nonsurgical approaches. often conducted on new treatments). Since the publication of this study, alleviation of symptoms has consistently been reported in studies of LIDR with ProDisc-L, including lower risk of adjacen

Following this research the International Society for the Advancement of Spine Surgery (ISASS) educated patients, health statement saying that LTDR represents

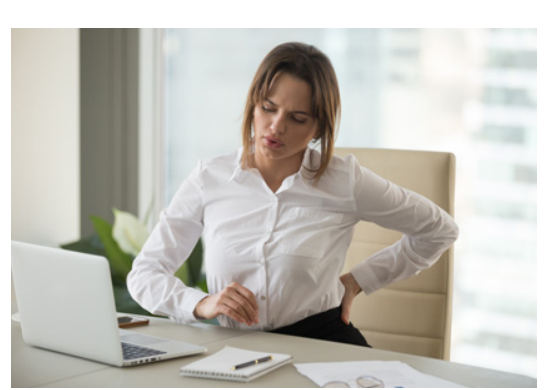

Lumbar disc degenerative disease causes
the discs to break down and can cause huge care providers, insurers and many others 

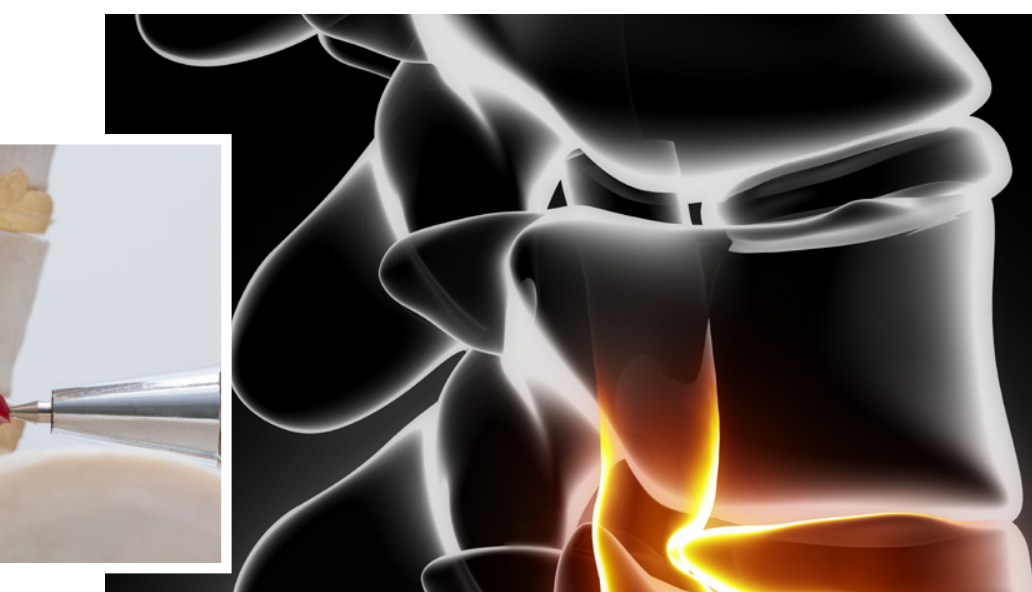

a proven technique and a well-tested treatment that must be accepted for the benefit of patients because it should complications than fusion.

\section{IT'S COMPLICATED...}

Despite this positive statement from been slow. This is largely durg to ITDR being an incredibly technically challeng procedure. Years of research has shown that optimum placement of the LAD is essential for successful outcomes. For example, the risk of developing facet arthrosis (limited spinal movement) increases in patients where the disc implan has not been positioned correctly. In the case of ProDisc-L, a study of 42 patients three years after surgery revealed that $37 \%$ exhibited signs of facet arthrosis verses $63 \%$ who did not. It was revealed that was the cause of facet arthrosis.

Although LTDR has some complications relating to incorrect positioning, far superior to fusio for the trot are of LDDD Chronic pain can cause disability; evidence shows improved physical capability following ProDisc-L implantation. It's unsurprising that successful LTDR has a positive impact
improves a patient's quality of life.

\section{...AND CHALLENGING}

LTDR has survived a difficult introduction to the spinal surgical community but it is now, albeit slowly, gaining acceptance. for by the logistic for by the logistical challenges faced by spinal surgeons whow
LTDR into their clinic.

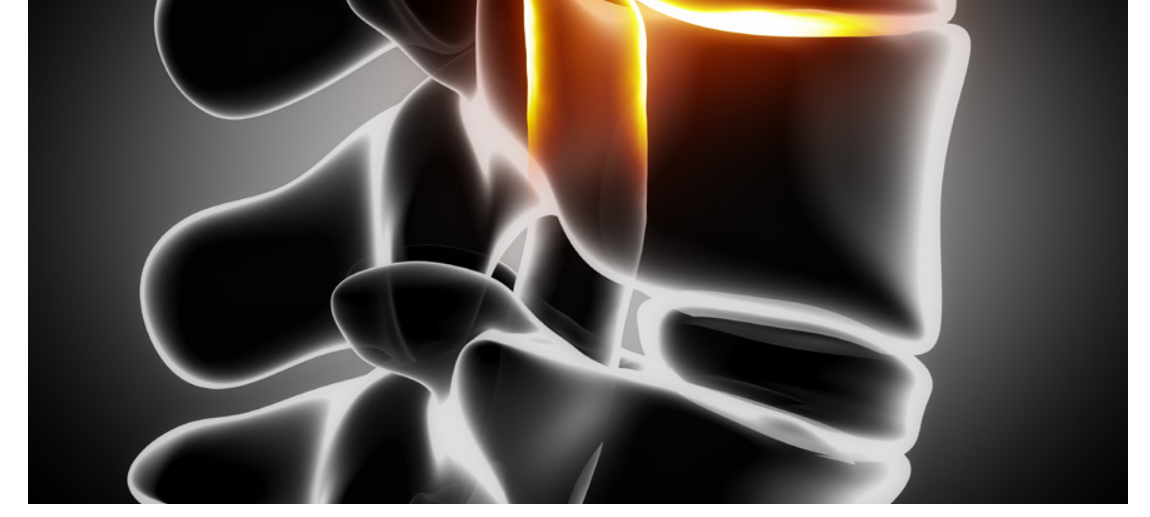

It's unsurprising that successful LTDR has a positive impact and improves a patient's quality of life.

Imagine you are a spinal surgeon wanting where does this leave patients? If a spina to introduce LTDR as a procedure in your surgeon proposes spinal fusion to a clinic. First, you must train in a centre patient with LDDD and fails to discuss of excellence for LTDR because its a new, other procedures such as LTDR, then challenging protocol, and you must ensure that surgeon is not fulfiling his/her duty

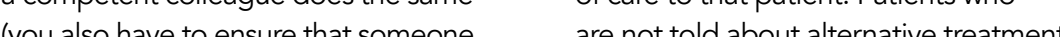
else is covering your work at the practice and are disatisfied with their fusion in the meantime). Before committing treatment are, rightly upset that they to this training, you should have received were not informed about LDR and its a commitment from local helth insurance proven benefits over fusion. It's important companies that they will support LTDR that patients are aware of all treatment (without this support, people will likely options so an informed decision can not choose the surgery as it becomes be made. Surgeons must advocate for too expensive, then your training will their patients, regardless of their opinion become redundant). Bearing in mind of LTDR, and they must therefore discuss the FDA has only approved LTDR for the the concept of the procedure for patients replacement of a single disc, this must also be adhered to unless you persuade the FDA to alter its approval. This a long, the FDA to alter its approval. This a long,
difficult and costly process for surgeons
superiority the wer fusion and discectom AWARENESS procedure on board at their clinics,
If surgeons are struggling to take this

\section{Behind the Research}

\section{Dr Stephen Beatty}

\section{Research Objectives}

Professor Beatty's research interests include cataract and age-related macular degeneration.

\section{Detail}

Bio

Professor Stephen Beatty suffered unbearable pain

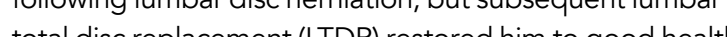
Peeved a his original spinal surgeon's filure to disclose the existence of LTDR, Stephen wishes to bring this proven and FDA-approved technology to the attention of patients.

\section{References}

Beatty, S. (2018). We Need to Talk about Lumbar Total Disc Replacement. International Journal of Spine Surgery, 12 (2) 201-240. https://www.ijssurgery.com/content/12/2/201.

\section{Personal Response} If awareness of LTDR continues to increase,
how long do you think it will be before LTDR is a standard treatment?

II Like all innovations, consumer-led demand is key
to success. In other words, if patients are aware of LTDR the spinal surgical community will respond

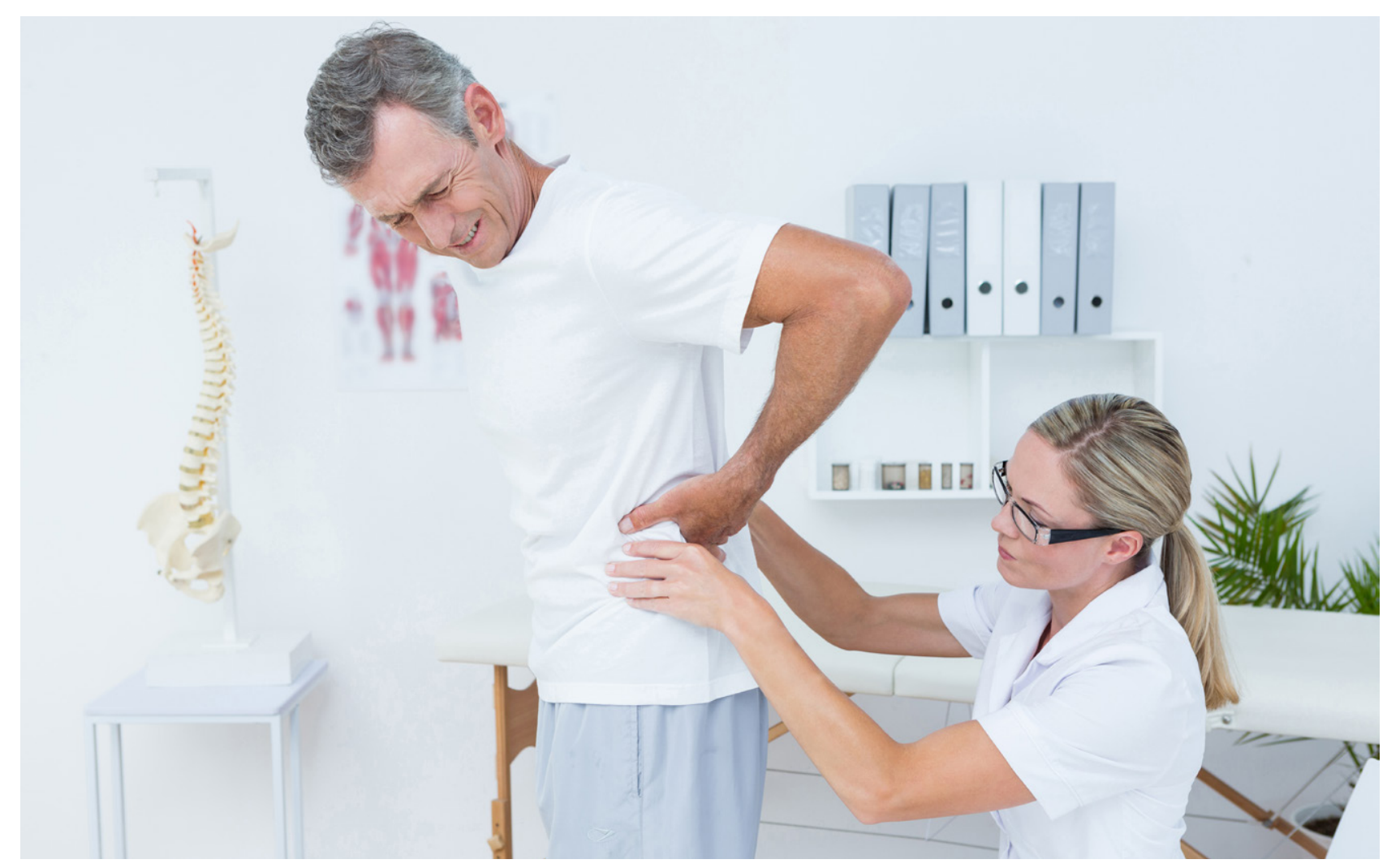

